\title{
PHOTOGRAMMETRIC TECHNIQUES FOR PALEOANTHROPOLOGICAL OBJECTS PRESERVING AND STUDYING
}

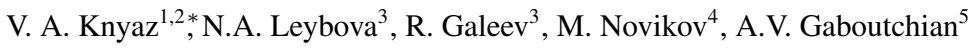 \\ ${ }^{1}$ State Research Institute of Aviation System (GosNIIAS), 125319 Moscow, Russia - knyaz@gosniias.ru \\ ${ }^{2}$ Moscow Institute of Physics and Technology (MIPT), Russia \\ ${ }^{3}$ Institute of Ethnology and Anthropology RAS, Moscow, Russia - nsuvorova@mail.ru \\ ${ }^{4}$ Research Center Crystallography and Photonics RAS, Shatura, Russia - novikov@ rambler.ru \\ ${ }^{5}$ Moscow State Medical-Stomatological University, Moscow, Russia - armengaboutchian@ mail.ru
}

\section{Commission II, WG II/10}

KEY WORDS: Fossil, Accuracy, Automation, 3D model, Palaeoanthropology, Non-contact measurements

\begin{abstract}
:
Paleo-anthropological research has its specificity closely related with studied objects. Their complicated shape arises from anatomical features of human skull and other skeletal bones. The degree of preservation is associated with the fragility of palaeo-anthropological material which usually has high historical and scientific value. The circumstances mentioned above enhance the relevance of photogrammetry implementation in anthropological studies. Thus, such combination of scientific methodologies with up-to-date technology creates a potential for improvement of various stages of palaeo-anthropological studies. This can be referred to accurate documenting of anthropological material and creation of databases accessible for wide range of users, predominantly research scientists and students; preservation of highly valuable samples and possibility of sharing information as 3D images or printed copies, improving co-operation of scientists world-wide; potential for replication of contact anthropometric studies on 3D images or printed copies providing for development of new biometric methods, and etc. This paper presents an approach based on photogrammetric techniques and non-contact measurements, providing technological and methodological development of paleo-anthropological studies, including data capturing, processing and representing.
\end{abstract}

\section{INTRODUCTION}

The important part of information needed for paleoanthropological study comes from geometric measurements of skulls and skeletal bones. Measurements and analysis of linear, angular and shape parameters allow to make decisions on paleoanthropological characteristics of an object. A set of standard landmark points is used for the analysis, these points reflecting anatomical features of an object.

Special mechanical instruments are designed for performing geometric measurements. They allows carrying out required measurements in accordance with standardized methods. Manual measurements are still widely applied in a practice of paleoanthropological studies. The main tools of an anthropologist are precise mechanical instruments such as a ruler and various types of calipers. Also an expert has a possibility to apply more complicated mechanical instruments such as sliding caliper (Martin type), coordinate caliper (Aichel type), spreading caliper, craniofor (Mollison type) and mandibulometer (Figure 1).

In some cases (such as tooth measurements, or odontometry) measurement landmarks are unattainable because of impossibility to access the required landmark by a calliper or due to object fragility. So practice of manual measurement significantly restricts the possibility of gathering statistically reliable data and sharing it for wide and comprehensive research.

Digital imaging techniques create new possibilities for paleoanthropological studies. They provide more information of higher

\footnotetext{
${ }^{*}$ Corresponding author
}

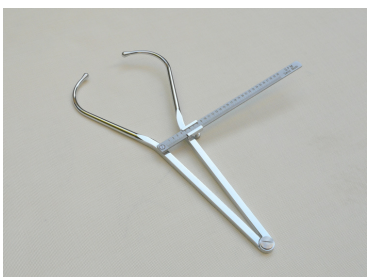

(a) Spreading Caliper

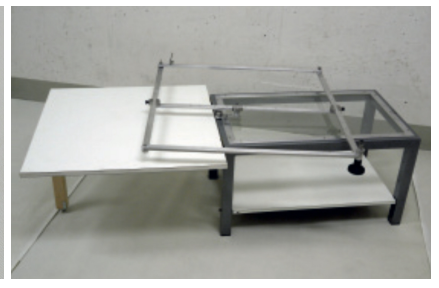

(b) Rectangular dioptrograph
Figure 1. Anthropological mechanical instruments

value and comprehensiveness, contributing to informativeness enhancement of paleoanthropological studies. Computed tomography, laser scanning, thermal imaging are being applied in such fields as anthropology, archaeology, forensic science. These techniques produce new types of data such as images of object sections, surface 3D models, voxel 3D models, textured 3D models of studied objects.

Photogrammetry has some advantages for paleoanthropological analysis due to non-contact principle of measurements, high accuracy of measurements, possibility of flexible adjustment of working space and resolution, possibility of accurate photorealistic texture mapping. Photorealistic 3D models of paleoanthropological object can be easily shared and used for public visual and morphometric study.

Paleoanthropology imposes specific requirements to produced 3D model (Weber, 2015). Along with accuracy and given level of de- 
tails paleo-anthropological 3D models have to be accurately textured with a high resolution. In addition oftentimes 3D models with varying levels of details in different parts of the model become necessary (Subsol et al., 2002), (Knyaz et al., 2017).

The paper presents photogrammetric techniques for creating, photorealistic texturing and automated morphometric study of 3D models of paleoanthropological objects.

\section{RELATED WORK}

Application of digital imaging techniques to paleoanthropological studies has started with the first imaging systems (Hounsfield, 1973). An impressive progress and increasing availability of such systems provides for greater than ever number of products and possible applications. The feasibility of studying 3D model rather than the object itself creates conditions for preserving of high value material. Another noticeable advantage of such techniques is in repeatability of studies and possibility of implying different methods of research. The detailed review of applications of computed tomography in paleoanthropology is presented in in (Uldin, 2017).

Landmark points obtained from 3D models are used for statistical analysis using as morphometric methods (Sokal and Rohlf, 1995) as geometric morphometrics (Bookstein, 1992), (Richtsmeier et al., 2002).

Studies of morphological variations in cranial landmark configurations between Neanderthals and anatomically modern Homo (Harvati, 2002), (Harvati, 2003) have showed that the morphological distance between these two groups is so large that the hypothesis that Neanderthals represent a subspecies of $\mathrm{H}$. sapiens cannot be supported.

A 3D model of a lower molar tooth was used to identify and quantify the different stages of tooth development (Smith et al., 2007). The model was produced by micro-computed tomography of human teeth that provides accurate quantification of the outer and inner enamel and dentin boundaries of individual cusps. The 3D model was used to compare morphogenesis in the lower second deciduous molar and lower first permanent molar.

A combination of virtual reconstruction and geometric morphometrics was studied in (Milella et al., 2015). Authors showed that such data offers new insights into the modularity and integration of pelvic ontogeny, while at the same time demonstrating the usefulness of a combined virtual reconstruction/geometric morphometrics approach as complement to classical methods of paleopathology.

Tooth crown morphology was studied in (Gaboutchian et al., 2017) using accurate teeth $3 \mathrm{D}$ models. The study evaluate new morphological features characterizing genetically conditioned tooth relief features averse to substantial changes under environmental factors during lifetime.

Some investigations were carried out for evaluating the reliability of measurements taken on the 3D digital models created by laser scanning comparing with directly taken linear measurements on dry skulls (Toneva et al., 2017), which allows assessing the good agreement between both measuring methods and applicability of laser scanning for paleoanthropological measurements.

\section{PHOTOGRAMMETRIC MEANS FOR ANTHROPOLOGICAL ANALYSIS}

Photogrammetric technique of 3D reconstruction has a distinctive advantage in comparison to methods laser scanning or computed tomography which consists in providing of two types of data - images of an object and its 3D model. These data has mutual relations arising from the principles of photogrammetric data processing.

Photogrammetric approach is developed for anthropological morphometric data acquiring, fusion, representing and processing. A special photogrammetric 3D scanning system is developed to meet particular anthropological requirements. It allows to acquire high resolution $3 \mathrm{D}$ and $2 \mathrm{D}$ data of different scales according to paleoanthropological needs.

\subsection{Photogrammetric system configuration}

3D models for paleoanthropological applications were obtained in automated mode on original photogrammetric system. It includes two digital high resolution cameras, a structured light projector, and a digital SLR camera for acquiring high resolution texture (Figure 2).

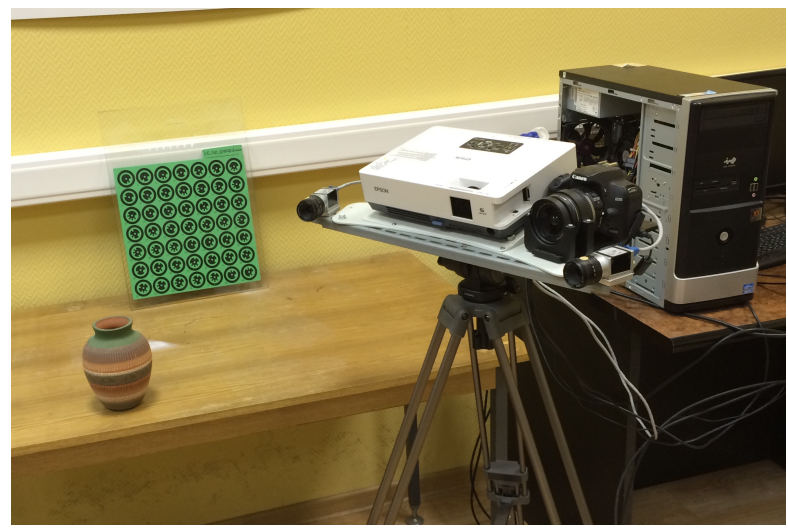

Figure 2. Photogrammetric system

The main specifications of the photogrammetric system are presented in Table 1 .

The photogrammetric system was developed to satisfy the needs of paleoanthropological researchers. Therefore it supports two configurations, the first one ("macro") being for 3D surface capturing in the working space of approximately $300 \mathrm{~mm} \times 300 \mathrm{~mm}$ x $300 \mathrm{~mm}$, and the second one ("micro") - for acquiring 3D data with higher level of detail with working space of about $80 \mathrm{~mm} \mathrm{x}$ $80 \mathrm{~mm}$ x $80 \mathrm{~mm}$.

The system allows producing high resolution 3D models (Figure 3 ) in automated mode. Original calibration procedure provides an accuracy of the generated 3D models at the level of $0.05 \mathrm{~mm}$ for the "macro" and $0.008 \mathrm{~mm}$ - for "micro" configuration respectively. This accuracy is quite sufficient for the task of anthropological analysis.

\subsection{Accurate texture mapping}

Textured 3D model provides more information for an expert because some features of interest can be precisely defined on color 


\begin{tabular}{||lr||}
\hline Camera & \multicolumn{1}{|c||}{ Basler acA1300-60gm } \\
\hline \hline Resolution & $1282 \mathrm{px} \times 1026 \mathrm{px}$ \\
\hline Sensor Size & $6.8 \mathrm{~mm} \times 5.4 \mathrm{~mm}$ \\
\hline Mono/ Color & Mono \\
\hline Shutter & Global \& Rolling Shutter \\
\hline Frame Rate & GigE \\
\hline Interface & Canon 1000D \\
\hline \hline DSLR camera & $3888 \mathrm{px} \times 2592 \mathrm{px}$ \\
\hline \hline Resolution & Color \\
\hline Sensor Size & USB 2.0 \\
\hline Mono/ Color & Epson EMP 1705 \\
\hline Interface & XGA, 1024 x 768, 4:3 \\
\hline \hline Projector & $400: 1$ \\
\hline \hline Resolution & \\
\hline Contrast Ratio & \\
\hline
\end{tabular}

Table 1. Main specifications of the photogrammetric system

images of studied objects. So the calibration of the photogrammetric system was performed for all three cameras using one calibration test field. This technique provides accurate texture mapping and accurate $3 \mathrm{D}$ coordinates measuring of the textured $3 \mathrm{D}$ model.

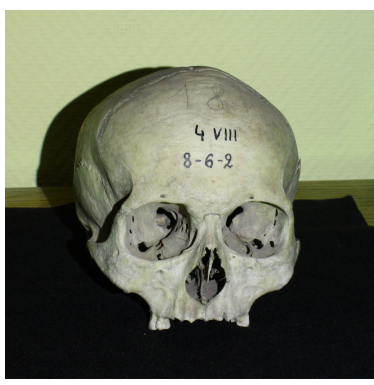

(a) Object image

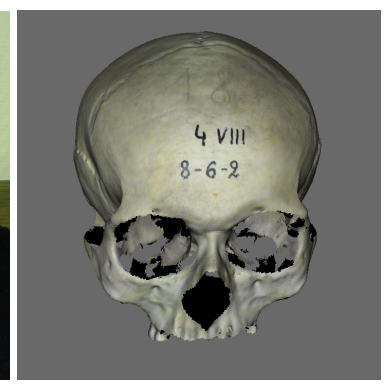

(b) 3D model

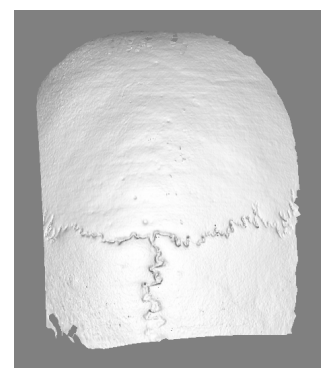

(c) 3D model fragment

Figure 3. An object and its textured 3D model

Figure 3 presents an image of a skull (a), its textured 3D model (b) and a fragment of the 3D model acquired with high resolution (c) ("micro" configuration of the photogrammetric system).

These 3D models can be used for such paleoanthropological studies as:

- measurements of morphometric anthropological parameters (distances, angles, coordinates)

- virtual and artistic reconstruction of an appearance basing on skull

- paleoanthropological restoration of objects

The applicability of structure-from-motion technique for geometric morphometrics study was estimated. 3D model of skull was reconstructed using Agisoft Photoscan software. The resulting $3 \mathrm{D}$ model is shown in Figure 4a. Figure 4b presents the comparison of this 3D model with the model produced by the photogrammetric system.

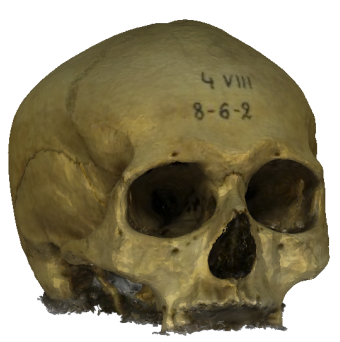

(a) SfM 3D model

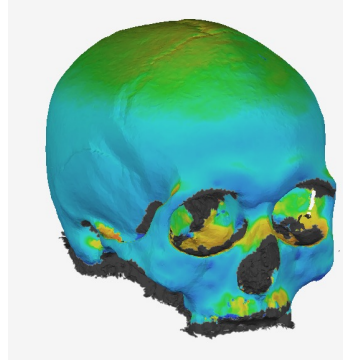

(b) Comparison with 3D scanning
Figure 4. SfM 3D model and comparison with 3D scanning

The standard deviation for surface difference is $1.22 \mathrm{~mm}$.

\section{AUTOMATED ANTHROPOLOGICAL MEASUREMENTS}

The main anthropological parameters needed for anthropological craniometric and morphometric analysis are:

- basic linear parameters (longitudinal $d_{\text {long }}$, transverse $d_{t r}$, height $d_{\text {height }}$ diameters)

- some height or depth parameters (canine fossa depth $h_{c f}$, height of forehead $\operatorname{arch} h_{f a}$, etc.)

- some characteristics of horizontal and vertical skull profiles (minimal distances along the skull surface between two landmarks, e.g. forehead arch $A_{\text {forehead }}$ )

- a set of angular parameters (forehead angle $\alpha_{\text {forehead }}$, nose bones angle $\alpha_{\text {nose }}$, etc. )

The most of anthropological measurements are carried out in standard skull position defined by sagittal plane and Frankfurt horizontal. So the first step for automated measurements is transforming 3D model in the standard system of coordinates basing on a set of landmarks.

\subsection{Automated measurements}

A set of algorithms for automated landmarks detection and recognition is proposed as a substitution of the traditional technique. They use both object 3D model and high resolution 2D texture images for recognition of required landmarks.

To evaluate the accuracy and convenience of using 3D models for morphometric measurement a set of accurate 3D models of paleoanthropological objects was generated. Then given series of standard morphometric measurements were made by traditional (manual) and the automated techniques (Figure 5). 


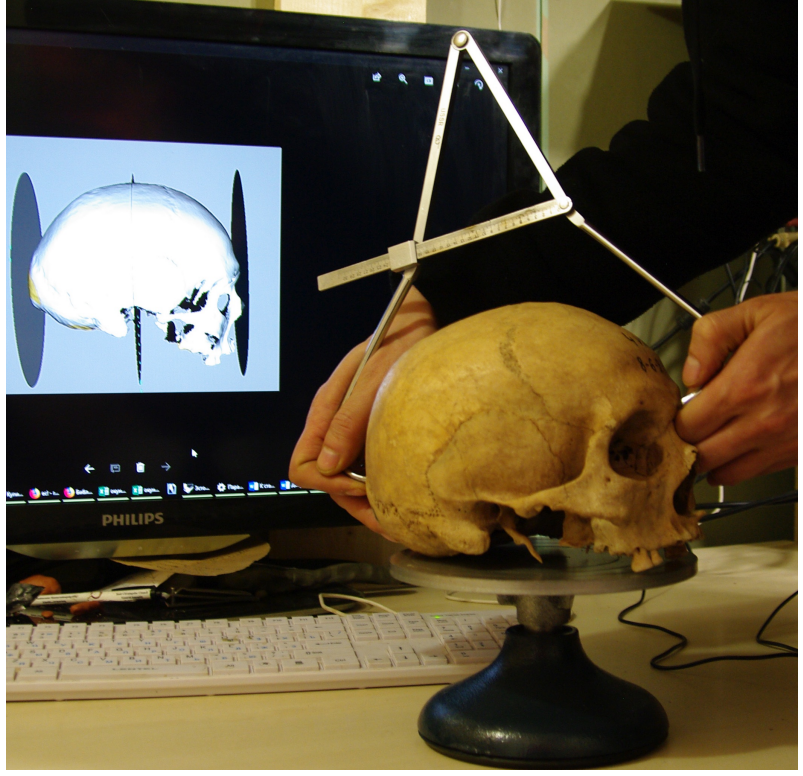

Figure 5. Anthropological parameters measuring

\subsection{Manual measurements}

The manual measurements were performed by an anthropologist using standard techniques (Bookstein, 1992). The results of manual measurements are presented in Table 2 in comparison with anthropological parameters measured on 3D model in manual and automated modes.

\begin{tabular}{|clccc|}
\hline$\#$ & Parameter & manual & 3D manual & 3D automatic \\
\hline 1 & $d_{\text {long }}, m m$ & 197 & 197.3 & 197.2 \\
\hline 8 & $d_{\text {tr }}, m m$ & 154 & 154.1 & 154.5 \\
\hline 9 & $w_{\text {min }}^{\text {foread }}, m m$ & 131 & 131.3 & 131.0 \\
\hline 10 & $w_{\text {max }}^{\text {foread }}, m m$ & 136 & 136.2 & 135.9 \\
\hline 17 & $d_{\text {height }}, m m$ & 119 & 119.2 & 118.9 \\
\hline & $h_{\text {cf }}, m m$ & 4.0 & 3.9 & 4.0 \\
\hline & $h_{\text {fa }}, m m$ & 23 & 23.1 & 23.1 \\
\hline 32 & $\alpha_{\text {forehead }},{ }^{\circ}$ & 82 & 82.4 & 81.6 \\
\hline 72 & $\alpha_{\text {profile }}{ }^{\circ}$ & 95 & 94.7 & 95.3 \\
\hline 75 & $\alpha_{\text {nose }}{ }^{\circ}$ & 53 & 52.5 & 52.9 \\
\hline
\end{tabular}

Table 2. Comparison of manual an automated measurements

Table 2 shows that the 3D models obtained by the developed photogrammetric system can be applied for geometric morphometric analysis as in manual as in automated mode. The accuracy of the geometric parameters determination on a 3D model is high enough for anthropological study.

Contours of scull were estimated by use of dioptrograph (Martin type) in two views (full face and profile), and the obtained data served for comparison with similar parameters obtained on 3D model. The contour comparison proves required accuracy and resolution of produced 3D models.

\subsection{Morphological analysis}

Machine learning based technique for automated recognition of tooth morphological type is proposed. It corresponds to existing classifications (Figure 6) and uses calculations of curvatures and digital elevation model of tooth 3D models.

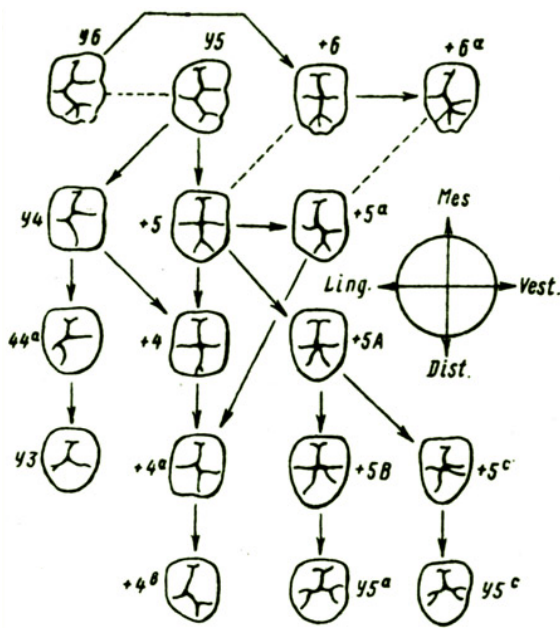

Figure 6. Tooth type classification

A dataset of tooth 3D models is used to prepare data for machine learning. The dataset includes 308 tooth 3D models acquired by the photogrammetric system. Five classes of teeth are presented in the 3D models dataset. Tooth 3D model serves for creating curvature image (Figure 7(a)) and a depth image (Figure 7(b)) of an occlusal surface of a tooth. These images were collected in two datasets (curvature and depth datasets) for deep learning. For dataset augmentation a set of such images was generated for each tooth $3 \mathrm{D}$ model by varying the orientation and processing of the 3D model.
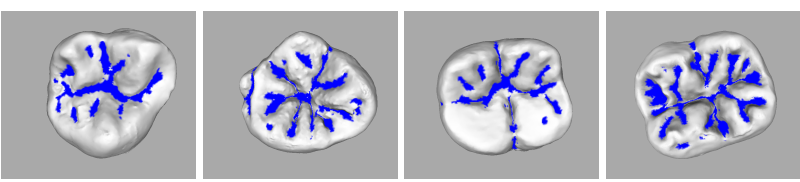

(a) Curvature images
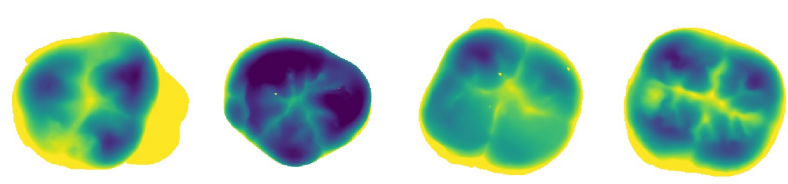

(b) Depth images

Figure 7. Samples from training datasets

The resulting image dataset contains 11088 images as for curvature as for depth signature. Each dataset of tooth images was splitted up into training set (9360 images) and testing set (1728 images). ResNet-50 (He et al., 2016) deep convolutional neural network was used for training.

Precision and recall criteria are calculated for evaluation of recognition quality defined as:

Positive predictive value or precision:

$$
P=\frac{N_{t r}^{k}}{N_{r}^{k}}
$$

and true positive rate or recall of : 


$$
R=\frac{N_{t r}^{k}}{N^{k}}
$$

where

$N_{t r}^{k}$ - true positive number (number of true recognized objects) for class $k, k=1, . ., K$;

$K$ - number of classes in dataset;

$N_{r}^{k}$ - number of all (true positive+false positive) objects recognized as an object of the class $k$;

$N^{k}$ - total number (true positive+false negative) of objects of the class $k$ in a dataset.

Both morphometric signatures (curvature and depth) demonstrated high scores (above 0.95 ) on testing dataset. The estimation of recognition quality on testing dataset showed better results $\left(P_{c}=\right.$ $\left.0.97, R_{c}=0.98\right)$ for curvature signature. These results can be overestimated because of relatively small dataset volume. The performance will be estimated on extended dataset as it accumulate new 3D models.

\section{STEREOLITHOGRAPHY RECONSTRUCTION}

Another advantage of implementation of object 3D models in a practice of paleoanthropological researches is a possibility of producing accurate hard copies by additive technologies. These hard copies of paleoanthropological objects could be used artistic reconstruction appearance basing on a given skull, for creating reference 3D collections and databases for valuable data exchange.

In current research hard copies of obtained paleoanthropological 3D models were produced by stereolithography (SLA) 3D printing technology (Figure 8).

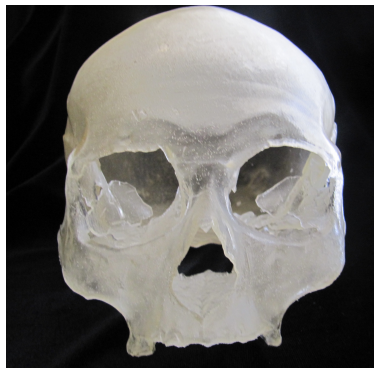

(a) SLA copy

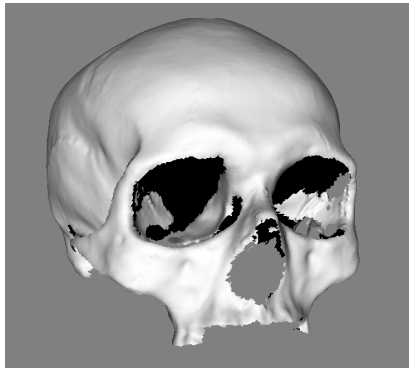

(b) 3D model of SLA copy
Figure 8. SLA copy of a skull and its 3D model

The main objective of producing hard copies of the obtained 3D models is in verifying the quality and accuracy of 3D printing and in estimating the possibility of 3D SLA models using in paleoanthropological studies during educating process or scientific research. Stereolithographic 3D models were measured manually by an anthropologist and using the photogrammetric system. Accuracy estimation results show applicability of stereolithographic reconstruction for procedures of appearance reconstruction and object restoration in anthropology. For procedures requiring higher degree of precision, e.g. odontometry, the accuracy of SLA 3D copies is insufficient.

The stereolithography model was scanned by the photogrammetric system. The obtained 3D model was compared with 3D model

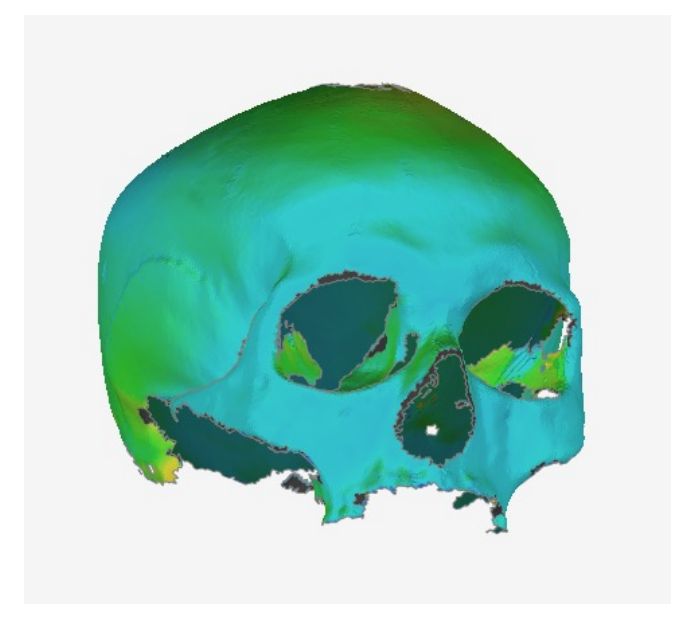

Figure 9. Comparison of SLA copy with parent 3D model

of the object used for SLA copy producing. The results of comparison of these two models is shown in Figure 9. The standard deviation for surface difference is $1.76 \mathrm{~mm}$.

\section{CONCLUSION}

Photogrammetric techniques for paleo-anthropological data capturing, processing, and representing are developed. Digital reconstruction of paleoanthropological 3D models provides accurate and photorealistic 3D and 2D data.

Automation of paleoanthropological analysis is achieved through application of proposed algorithms for landmarks recognition and morphometric analysis, which replicate traditional techniques.

To evaluate the accuracy and convenience of using 3D models for morphometric measurement several accurate 3D models of paleoanthropological objects were generated. Then given series of standard morphometric measurements were made by traditional (manual) and automated techniques. The comparison of measurement results shows good correspondence, which verifies the correctness of the developed algorithms.

Deep learning approach demonstrated high performance for task of tooth morphology recognition on data produced from photogrammetric tooth $3 \mathrm{D}$ models.

The applicability of using stereolithographic 3D copies of paleoanthropological objects for researches and sharing rare data was studied. Estimation of an accuracy of produced stereolithography 3D models shows that they can be successfully used for educational purposes and for tasks of appearance reconstruction.

\section{ACKNOWLEDGEMENTS}

The work was performed with the support by Grant 17-29-04509 of Russian Foundation for Basic Research (RFBR).

\section{REFERENCES}

Bookstein, F. L., 1992. Morphometric Tools for Landmark Data: Geometry and Biology. Cambridge University Press. 
Gaboutchian, A. V., Knyaz, V. A. and Leybova, N. A., 2017. Measurements of cuspal slope inclination angles in palaeoanthropological applications. ISPRS - International Archives of the Photogrammetry, Remote Sensing and Spatial Information Sciences XLII-2/W4, pp. 185-191.

Harvati, K., 2002. Models of shape variation between and within species and the neanderthal taxonomic position: A $3 \mathrm{~d}$ geometric morphometrics approach based on temporal bone morphology. three-dimensional imaging in paleoanthropology and prehistoric archaeology. Proceedings of the XIVth UISPP Congress 120, pp. $25-30$.

Harvati, K., 2003. Quantitative analysis of neanderthal temporal bone morphology using three-dimensional geometric morphometrics. American Journal of Physical Anthropology pp. 323338 .

He, K., Zhang, X., Ren, S. and Sun, J., 2016. Deep residual learning for image recognition. 2016 IEEE Conference on Computer Vision and Pattern Recognition (CVPR) pp. 770-778.

Hounsfield, G. N., 1973. Computerized transverse axial scanning (tomography): Part 1. description of system. The British Journal of Radiology 46(552), pp. 1016-1022. PMID: 4757352.

Knyaz, V. A., Zheltov, S. Y. and Chibunichev, A., 2017. Photogrammetric techniques for analysis and visualization of changes in $2 \mathrm{~d}$ and $3 \mathrm{~d}$ data: Plastic surgery application. ISPRS - International Archives of the Photogrammetry, Remote Sensing and Spatial Information Sciences XLII-2/W4, pp. 109-114.

Milella, M., Zollikofer, C. P. and de Len, M. S. P., 2015. Virtual reconstruction and geometric morphometrics as tools for paleopathology: A new approach to study rare developmental disorders of the skeleton. The Anatomical Record 298(2), pp. 335345 .

Richtsmeier, J. T., Deleon, V. B. and Lele, S. R., 2002. The promise of geometric morphometrics. American Journal of Physical Anthropology 119(35), pp. 63-91.

Smith, P., Müller, R., Gabet, Y. and Avishai, G., 2007. A computerized model for reconstruction of dental ontogeny: A new tool for studying evolutionary trends in the dentition. Springer Netherlands, Dordrecht, pp. 275-288.

Sokal, R. and Rohlf, F., 1995. Biometry: The Principles and Practice of Statistics in Biological Research. W.H. Freeman and Co.

Subsol, G., Mafart, B., Silvestre, A. and De Lumley, M., 2002. 3D Image Processing for the Study of the Evolution of the Shape of the Human Skull: Presentation of the Tools and Preliminary Results. In: B. Mafart, H. Delingette and G. Subsol (eds), ThreeDimensional Imaging in Paleoanthropology and Prehistoric Archaeology, BAR International Series 1049, pp. 37-45.

Toneva, D., Nikolova, S., Georgiev, I. and Tchorbadjieff, A., 2017. Accuracy of Linear Craniometric Measurements Obtained from Laser Scanning Created 3D Models of Dry Skulls. Springer International Publishing, Cham, pp. 215-229.

Uldin, T., 2017. Virtual anthropologya brief review of the literature and history of computed tomography. Forensic Sciences Research 2(4), pp. 165-173.

Weber, G. W., 2015. Virtual Anthropology and Biomechanics. Springer Berlin Heidelberg, Berlin, Heidelberg, pp. 937-968. 